\title{
Reliable Design and Implementation of Wired Headphone
}

\author{
Liu Guo-qing
}

\author{
Wenzhou Vocational \& Technical College, Wenzhou. 325035 \\ 3685424@qq.com
}

Keywords: Reliability, Implementation,Design,Headphone.

\begin{abstract}
This project mainly aims at the students which is convenient, simple and easy to carry. In addition, it basically has no harm to our ears, and we will not feel dis-comfortable after using for a long time, it is suitable for games, movies, music, voice and so on. We can also carry it to other places and use for games which is easy and comfortable. In terms of the shape, it combines the shape of traditional headphone as well as the new telescopic ladder shape, all these make the whole product have its own characteristics. When using together with the current headphone, the users may enjoy the special sound effect better. Meanwhile, we may also promote to use it in E-sports games and make it be the priority selection of all the travel enthusiasts.
\end{abstract}

\section{Introduction}

Under the background of social development, this design analyzes psychological and physiological demands of various groups more deeply through analyzing and researching information process, importance as well as development course of using earplug type headphone, and combining with ergonomics, design psychology, humanized design and other related theories of industrial design. Computer is essential in the era of information. And with the emergence of notebook, computer is no longer fixed: office workers can go to the office with computers, students can take to dormitory and we can also take our computers when playing. This time, a lot of people like to have a earphone or headset, and compared with headset, the headphone is convenient to carry. While the ear packs are more soft and you will feel more comfortable, headset also has the same functions with microphone, and we can purchase different headsets according to different situations.

\section{Domestic and foreign development situation}

The development of foreign headset focuses on comfort and feeling, it starts from the feeling of users and allows them experience various feelings. Later I will take some well-known brands aboard for example:

Sennheiser is the most famous brand in Europe and it has become the representative of European headset. It has the following features: not exaggerate, its sound is more real and suitable for long-time listening which will not bore you. On the contrary, the voice of Japanese headset is more exaggerated and the sound pollution is serious, and we will feel flustered after listening for a while. Headset from Sennheiser can make you have a correct understanding of the music.

Gerd is also a famous headset brand aboard and it can be divided into MB QUART and GRADO, and both of them are famous headset brands. While although MB QUART has fewer product models and the price of its high-end products is less than two thousand yuan, its voice is really unique, especially for symphony orchestra as well as fever girls. It receives a quite good evaluation from headset fanciers. Through the analysis above, we can find that the design emphasis of foreign headset is the comfort and the enjoyment on sound effect. This project regards foreign quality as the target to conduct design. 


\section{Headset situation}

The current headset is mainly designed for computer which is a kind of personal enjoyment without audio, it is mainly used for home desktops and laptops. The headset also has different types: some are relatively small and easy to carry; some have big ear packs and are very comfortable; some cab be folded to reduce occupied space and some are telescopic and suitable for different people.

Smaller headset is usually used in notebooks and it can be put in the computer bag to carry because of its smaller design. Most of theme are wire headset because its stability is relatively high, we only need to insert the plugs of earpiece and microphone when using. The operation is more convenient, and after usage, we need to maintain the relative position of earpiece and microphone. Under normal circumstances, winding the wire around McCarthy may relatively reduce a little space. While it also has own disadvantages, such headset receiver is very small and thin, and our ears will feel very painful and uncomfortable after using for a long time. In addition, its quality is not very good.

Headset with relatively large ear packs is mainly used at home or used for basically fixed notebooks. McCarthy is relatively large because of large ear packs, and in most cases, it is used in fixed places. While this headset is very comfortable to wear and we will not feel painful. In addition, it is very thick and soft, the wire headset is more stable and we only need to place back after sorting.

Of course, there are still some portable folding headset as well as wireless headset. Folding headset may reduce space through the structures of ear packs and McCarthy and make it be portable under the case of occupying less space. While it has the same disadvantage with small headset: both of them are not suitable for long time use. While wireless headset is very few in the market, and most of them are used as radios in examination.

\section{Design objectives}

To solve the convenience and comfort of current headset, I design a retractable earphone. Most of the convenient headsets are folding, although they are easy to carry, they still lacks comfort. While the comfort headset with big ears package has a lack of convenience. As a result, I design a retractable earphone with big ears package, it has large and thick ear packs to comfort our ears when using. Because the big ears package occupy a large space, I design a telescopic device and the whole McCarthy is retractable, and the two ear packs can be combined together after shrinking. Then, the space occupied by headset reduces greatly. In addition, it presents a more regular shape which is easy to take, and it is especially suitable for young people like travel and games. It is convenient and reliable and suitable for most people. Apart from these, it can easily attract your attentions.

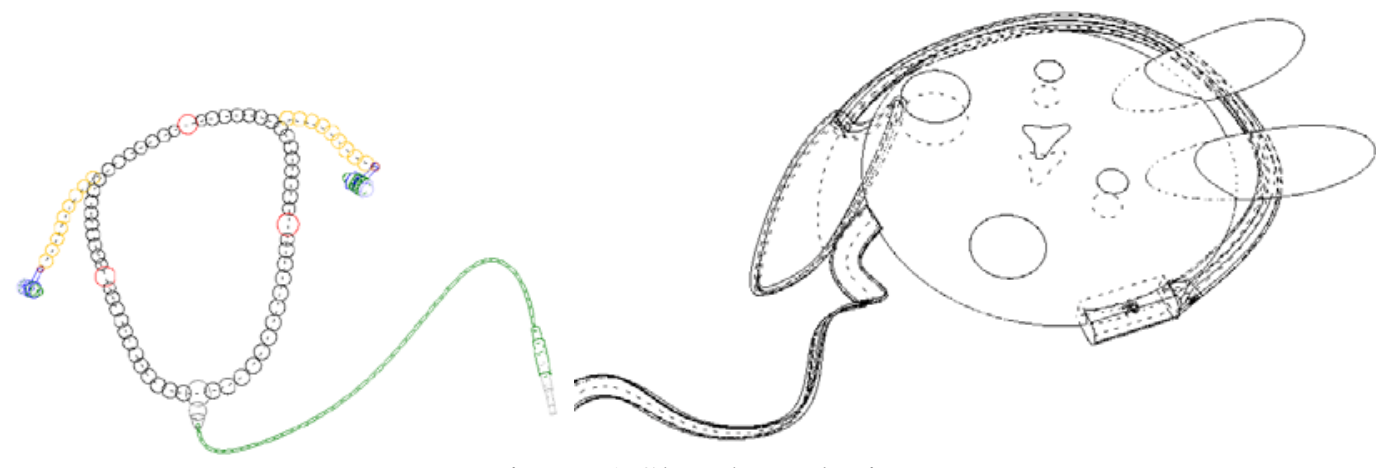

Figure 1 Sketch analysis

\section{Design positioning}

This portable and comfortable headset which is used in holiday, games, video, music is suitable for most students. In nowadays, the market for students is very large, such headset can attract their eyes and the functions are also very suitable, and it will surely become a mainstream product. 


\subsection{Modeling conceptual design of portable headset}

Product modeling bases on the traditional headset and makes out a more comfortable and convenient headset through changing part structure. Telescopic design of McCarthy not only solves the problem of reducing the occupied space to achieve convenient carrying, but also reflecting a mechanical sense. In addition, the black increases its decorous feeling and makes it look more cool on the whole which is more attractive. And the market demand for products also increases, the modeling design is shown in figure 2 .

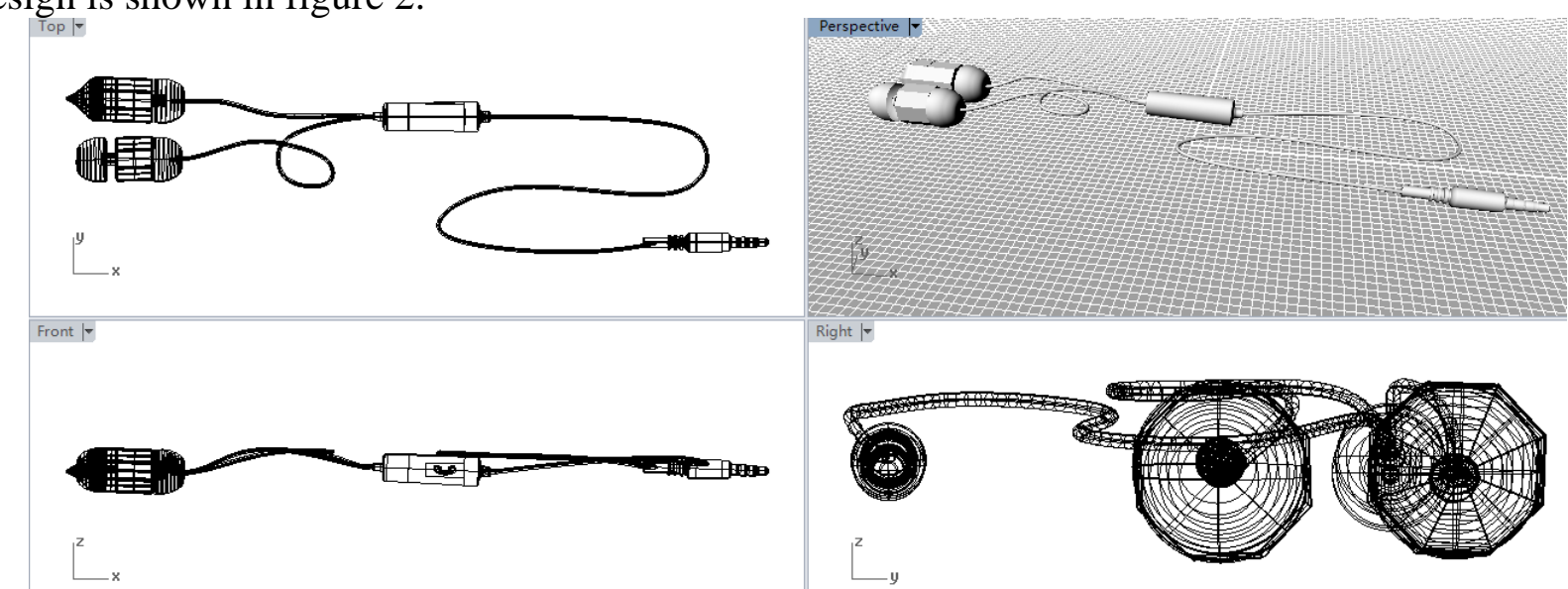

Figure 2 modeling design

\subsection{Conceptual design of microphone and USB}

Microphone design of this product gets rid of the swing type microphone and there is a fixed one under the receiver. Microphone is designed to be very small and it almost does not occupy space for convenient carrying. While it will not weaken the speech effect, and on the other hand, receiving intensity of such microphone is relatively huge, we can clearly say what we want to express without loud speaking. Apart from these, the listening will be very clear too and it has no effect on speaking if the mouth was far away. USB design aims at getting rid of the winding trouble of traditional cable ones which often look very messy because the wire must be wound up. Design of this USB solves this problem, USB device is just placed on one side of the headset, we may pull it out when not using, it is easy to understand. In addition, it is not easy to lose because we have to place it back on the headset.

The color is basically monochrome, and as shown in figure 1 , the wearing ways might be earplugs or headphones.

Table 1

\begin{tabular}{lllll}
\hline Product name & Color category & Wearing mode & $\begin{array}{l}\text { Whether there is } \\
\text { a microphone }\end{array}$ & $\begin{array}{l}\text { Headset plug } \\
\text { type }\end{array}$ \\
\hline Earphone mic & $\begin{array}{l}\text { Black, red and } \\
\text { white }\end{array}$ & $\begin{array}{c}\text { Earplugs, } \\
\text { headphones }\end{array}$ & None & Full-sized \\
\hline
\end{tabular}

\section{Features and innovations}

The design style of this headset is unique, it has the features of traditional headset as well as novel features and design, followings are the features and innovation points of this product:

Ladder shaped McCarthy design makes it has a mechanical sense. McCarthy uses the gradations of this ladder to expand and contract and achieve the purpose of reducing occupancy space.

Package size is shown in figure 3. It is inspired by the cartoon and meet all the defined requirements, and the innovation points are reflected in modeling and storage mode. The storage function of headset is achieved through the rotation of gears inside. 


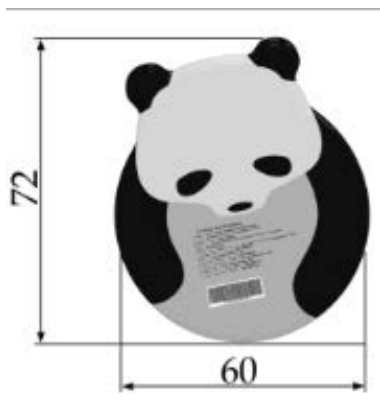

Figure 3 Size

The receiver loop uses plastic and rubber materials which not only protect the receiver, but also makes our ears more comfortable in usage. Figure 4 below shows the curving effect simulated by computer.

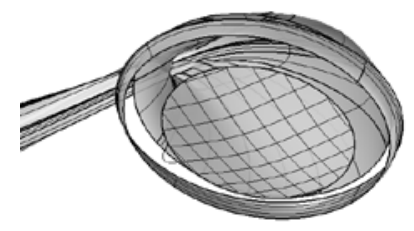

Figure 4 Computer-aided effect

The design of USB solves the situation that the traditional headset line will mess up and achieves the use within further distance. In addition, the plug placed beside handset has no influence on the whole modeling.

Microphone is very compact, it occupies a small space without affecting the use. A small microphone placed under the big one has a unique beauty on the appearance.

\section{Summary}

Most traditional headsets link a large McCarthy to two headsets and plus a mike as well as a long line, and this gives a feeling of confusion. Apart from this, the large headset also occupies a large space. As a result, a convenient headset which is easy to carry is necessary. In order to solve the above problems, it can be connected through USB cable and moves away the interference of the cable when using and shrinks to the size of two ear packets through McCarthy expansion to save space and solves the issues of most people who want to take it out. Meanwhile, this paper also proves the feasibility of portable earplugs. I also design a headset which occupies a small space and is easy to carry out to office, Internet cafes and games, etc. to meet the demands of various people, and last I make a brief assessment on the 3D model.

\section{References}

[1] GAO Li-wen.The Function of Packaging Optimum Design in Modern Logistics[J].China Logistics \& Purchasing, 2012 (5) : 58.

[2] PENG Guo-xun.The Packaging Design of Logistic Transport[M].Beijing: Printing Industry Press, 2006.

[3] ZHANG Yun-bo, PENG Guo-xun.Optimum Design of CushionPackaging for Twenty-six Inches LCD[J].Packaging Engineering, 2008, 29 (6) : 11-14.

[4] YANG Qing-liang.Innovation is Born: Contact with TRIZ[M]. Chengdu: Sichuan University Press, 2006.

[5] CHEN Guo-qiang.Process and Method of Product Design[M].Beijing: Machinery Industry Press, 2011. 